\title{
MET amplification in metastatic colorectal cancer: an acquired response to EGFR inhibition, not a de novo phenomenon
}

\author{
Kanwal Raghav ${ }^{1}$, Van Morris' ${ }^{1}$, Chad Tang ${ }^{2}$, Pia Morelli ${ }^{1}$, Hesham M. Amin ${ }^{3}$, Ken \\ Chen $^{4}$, Ganiraju C. Manyam ${ }^{4}$, Bradley Broom ${ }^{4}$, Michael J. Overman ${ }^{1}$, Kenna Shaw ${ }^{5}$, \\ Funda Meric-Bernstam ${ }^{6}$, Dipen Maru ${ }^{3}$, David Menter ${ }^{1}$, Lee M. Ellis ${ }^{7}$, Cathy Eng ${ }^{1}$, David \\ Hong $^{6}$, Scott Kopetz ${ }^{1}$ \\ ${ }^{1}$ Department of Gastrointestinal Medical Oncology, The University of Texas MD Anderson Cancer Center, Houston, TX, USA \\ ${ }^{2}$ Department of Radiation Oncology, The University of Texas MD Anderson Cancer Center, Houston, TX, USA \\ ${ }^{3}$ Department of Hematopathology, The University of Texas MD Anderson Cancer Center, Houston, TX, USA \\ ${ }^{4}$ Department of Bioinformatics and Computational Biology, The University of Texas MD Anderson Cancer Center, Houston, TX, USA \\ ${ }^{5}$ Sheikh Khalifa Bin Zayed Al Nahyan Institute of Personalized Cancer Therapy, The University of Texas MD Anderson Cancer \\ Center, Houston, TX, USA \\ ${ }^{6}$ Department of Cancer Therapeutics, The University of Texas MD Anderson Cancer Center, Houston, TX, USA \\ ${ }^{7}$ Department of Surgical Oncology, The University of Texas MD Anderson Cancer Center, Houston, TX, USA
}

Correspondence to: Scott Kopetz, email: skopetz@mdanderson.org

Keywords: amplification, MET, circulating-free DNA, fluorescence in situ hybridization, colorectal cancer

Received: March 14, 2016

Accepted: May 05, 2016

Published: July 13, 2016

\section{ABSTRACT}

Background: MET amplification appears to be a predictive biomarker for MET inhibition. Prior studies reported a MET amplification rate of 9-18\% in metastatic colorectal cancer (MCRC) but do not differentiate increased gene copy numbers due to chromosomal level aberrations from focal gene amplifications. Validation of MET amplification rate in $\mathrm{MCRC}$ is critical to this field.

Results: In tumor tissue-based analyses, overall MET amplification rate was $1.7 \%$ (10/590). MET amplification was seen in 0/103 (0\%), 4/208 (1.9\%) and 6/279 (2.2\%) cases, in cohorts 1,2 and 3, respectively. Rate of MET amplification in cfDNA of cohort 4 patients refractory to anti-EGFR therapy $(n=53)$ was $22.6 \%(12 / 53)$ and was significantly higher compared to patients not exposed to anti-EGFR therapy $(p<0.001)$.

Materials and Methods: We analyzed MET amplification in mCRC $(n=795)$ using different methods across multiple cohorts. Cohort $1(n=103)$ and $2(n=208)$ included resected liver metastases and tumor biopsies, respectively, tested for MET amplification using fluorescence in-situ hybridization [amplification: MET/CEP7 ratio $\geq 2.0$ ]. Using another tissue-based approach, cohort $3(n=279)$ included tumor biopsies sequenced with HiSeq (Illumina) with full exome coverage for MET [amplification: $\geq 4$ copies identified by an in-house algorithm]. Using a blood-based approach by contrast, cohort 4 $(n=205)$ included patients in whom the full exome of MET in circulating-free DNA (cfDNA) was sequenced with HiSeq.

Conclusions: Contrary to prior reports, in this large cohort, MET amplification was a rare event in $\mathrm{mCRC}$ tissues. In plasma by stark contrast, MET amplification identified by cfDNA occurred in a sizable subset of patients that are refractory to anti-EGFR therapy. 


\section{INTRODUCTION}

MET (mesenchymal-epithelial transition factor) proto-oncogene on chromosome $7 \mathrm{q} 31$ encodes for a receptor tyrosine kinase (RTK) and regulates a variety of downstream signaling pathways that initiate gene expression involved in promoting tumor growth, survival, angiogenesis, invasion and metastases. $[1,2]$ Due to its critical role in cancer biology, inhibition of the MET pathway is being actively investigated in numerous clinical trials. [1-4] MET amplification appears to identify a subset of cancers, uniquely sensitive to MET inhibitors, both in vitro and in vivo. [2, 5-7] MET amplification also drives resistance to anti-epidermal growth factor receptor (EGFR) monoclonal antibodies in colorectal cancer. [8] Consequently, MET amplification could potentially serve as a useful predictive biomarker of MET inhibitor response in mCRC clinical trials. Unfortunately, limited data exists regarding the prevalence of MET amplification in mCRC. [9] The most cited report assessed MET amplification using a quantitative polymerase chain reaction (PCR)-based assay $(N=217)$ and described an amplification rate of $9 \%$ in primary lesions and $18 \%$ in liver metastases. [9] However, these PCR-based assays were unable to differentiate between increased copy numbers from chromosomal level aberrations from focal gene amplification as is evident from studies in gastric cancer. $[5,6]$.

In this study, we examined a large number samples from mCRC cases across multiple cohorts to identify the frequency of MET amplification as determined by different methodologies along with a novel exploratory determination of MET amplifications in circulating cellfree DNA.

\section{RESULTS}

\section{MET amplification in tumor tissue-based biopsies}

MET amplification was seen in $10(1.7 \%$; $95 \%$ CI: $0.01-3.14 \%$ ) of 590 tumor tissue biopsies tested by both FISH and sequencing. MET amplification using FISH was seen in 0/103 (0.0\%; 95\% CI: $0.00-4.32 \%)$ and $4 / 208(1.9 \%$; $95 \%$ CI: $0.58-5.01 \%)$ cases in cohorts 1 and 2, respectively (MET/CEP7 ratio: 2.0-7.7). MET amplification using sequencing was seen in 6/279 (2.2\%; 95\% CI: 0.01-4.72\%) (MET gene copy numbers (GCN): 4.0-6.7) (Table 1). There was no significant difference among proportion of MET amplification between different cohorts $(p=0.34)$, FISH and sequencing $(p=0.53)$ and primary $(3.2 \% ; 95 \%$ CI: $1.6-6.0 \%)$ and metastatic sites $(0.5 \%$; $95 \%$ CI: $0.0-3.3 \%)(p=0.097)$ (Figure 1A-1C). Mutations in TP53 gene were the most common concurrent mutations seen in these patients (Supplementary Table S1).

\section{MET amplification in blood-based biopsies (cfDNA)}

In cohort 4, 53 RAS wild-type patients had been previously treated with and had disease progression on anti-EGFR therapy prior to collection of plasma. MET amplification in this anti-EGFR therapy refractory cohort was detected on cfDNA in 12 (22.6\%; 95\% CI: $13.31-$ $35.67 \%$ ) cases (Table 1 ). This proportion was significantly higher compared to MET amplification seen in anti-EGFR naïve tumor tissue-based biopsies $(p<0.001)$ (Figure 2A). Furthermore, this rate was also significantly higher compared to the rate of MET amplification seen in cfDNA of either RAS mutated patients $(p<0.001)$ or RAS wildtype tumors without prior anti-EGFR antibody exposure $(p=0.018)$ (Figure 2B). No difference in rate of cfDNA MET amplification was evident with other intervening therapies (Supplementary Figure S1).

\section{DISCUSSION}

In this large cohort of mCRC patients, we failed to validate the high prevalence of MET amplification in tissue samples as reported in prior studies with either FISH or sequencing. [9] Contrary to these reports, we observed that MET amplification is rare (1-2\%) in mCRC (as opposed to $9-18 \%$ ) and is not different between primary and metastatic lesions. [9] Our findings are consistent with the somatic copy-number alteration data generated by The Cancer Genome Atlas (TCGA) wherein only 1 case of high-level MET amplification was seen in a total of 276 colorectal tumors. $[10,11]$ We therefore believe that this study more accurately represents the incidence of MET amplification in de novo mCRC.

Discrepancies between our study and others can be explained by the limitations of PCR-based assays in detecting copy number. Both gene amplification and polysomy can result in increased GCNs in tumors. Experience in breast cancer reveals these two phenomena to be mechanistically distinct. Cases with Chromosome-17 (site of HER2/neu gene) polysomy compared to focal gene amplification of HER2, have lower GCNs, lower level of HER2 protein expression, features more consistent with HER2 negative tumors and do not derive any benefit from HER2-targeted treatment. [12,13] Similar discrepancy in rate of MET gene amplification was also observed in both gastric and lung cancer. $[5,6]$.

Gain of $7 q$ without any focal amplifications in MET in the TCGA cohort further supports for our findings. [11] Likewise, in a study using real-time quantitative PCR with transcript normalization no focal high-grade amplification of MET was seen in 103 liver metastases in $\mathrm{mCRC}$ but low level chromosome 7 polysomy was seen in 22 (21\%) cases. [14] MET inhibition showed no effect on tumor growth on patient-derived xenografts chromosome 7 polysomy cases, irrespective of MET expression. [14] 
Table 1: MET amplification proportion in multiple cohorts of mCRC

\begin{tabular}{|c|c|c|c|c|c|c|c|}
\hline \multicolumn{8}{|c|}{ Study of Tissue Based Biopsies } \\
\hline Cohort & $\mathbf{N}$ & $\begin{array}{l}\text { Primary } \\
\text { Site }\end{array}$ & $\begin{array}{l}\text { Metastatic } \\
\text { Site }\end{array}$ & $\begin{array}{c}\text { Relationship of } \\
\text { Samples to Prior } \\
\text { Systemic Therapy }\end{array}$ & $\begin{array}{l}\text { Test Employed } \\
\text { for MET } \\
\text { Amplification }\end{array}$ & $\begin{array}{c}\text { Proportion } \\
\text { of MET } \\
\text { Amplified } \\
\text { Cases }\end{array}$ & $\begin{array}{c}\text { Rate of MET } \\
\text { Amplification } \\
95 \% \text { CI }\end{array}$ \\
\hline $1^{\mathrm{a}}$ & 103 & NA & 103 & Pre-treatment & FISH & $0(0.0 \%)$ & $0.0 \%-4.3 \%$ \\
\hline $2^{\mathrm{a}}$ & 208 & 130 & 75 & Pre-treatment & FISH & $4(1.9 \%)$ & $0.6 \%-5.0 \%$ \\
\hline $3^{\mathrm{a}}$ & 279 & 161 & 110 & Pre-treatment & Sequencing & $6(2.2 \%)$ & $0.9 \%-4.7 \%$ \\
\hline \multicolumn{8}{|c|}{ Study of Blood Based Biopsies } \\
\hline Cohort & $\mathbf{N}$ & $\begin{array}{l}\text { Sub } \\
\text { Cohort } \\
\text { N }\end{array}$ & RAS Status & $\begin{array}{c}\text { Prior Treatment } \\
\text { \& Progression } \\
\text { on Anti-EGFR } \\
\text { Therapy }\end{array}$ & $\begin{array}{l}\text { Test Employed } \\
\text { for MET } \\
\text { Amplification }\end{array}$ & $\begin{array}{c}\text { Proportion } \\
\text { of MET } \\
\text { Amplified } \\
\text { Cases }\end{array}$ & $\begin{array}{c}\text { Rate of MET } \\
\text { Amplification } \\
95 \% \text { CI }\end{array}$ \\
\hline \multirow{3}{*}{4} & \multirow{3}{*}{205} & $4 \mathrm{~A}(53)$ & RAS-WT & Yes & \multirow{3}{*}{ Sequencing } & $12(22.6 \%)$ & $13.3 \%-35.7 \%$ \\
\hline & & 4B (43) & RAS-WT & No & & $2(4.7 \%)$ & $0.4 \%-16.3 \%$ \\
\hline & & 4C (109) & RAS-Mut & NA & & $4(3.7 \%)$ & $1.1 \%-9.4 \%$ \\
\hline
\end{tabular}

Abbreviations: CI, confidence interval; EGFR, epidermal growth factor receptor; FISH, fluorescence $i n$-situ hybridization; $\mathrm{N}$, number of patients; NA, not applicable; Mut, mutated; PCR, polymerase chain reaction; WT, wild-type.

a. Cohort 1 has only liver metastases; Site of the biopsy was unknown in 3 and 8 cases in Cohorts 2 and 3, respectively.
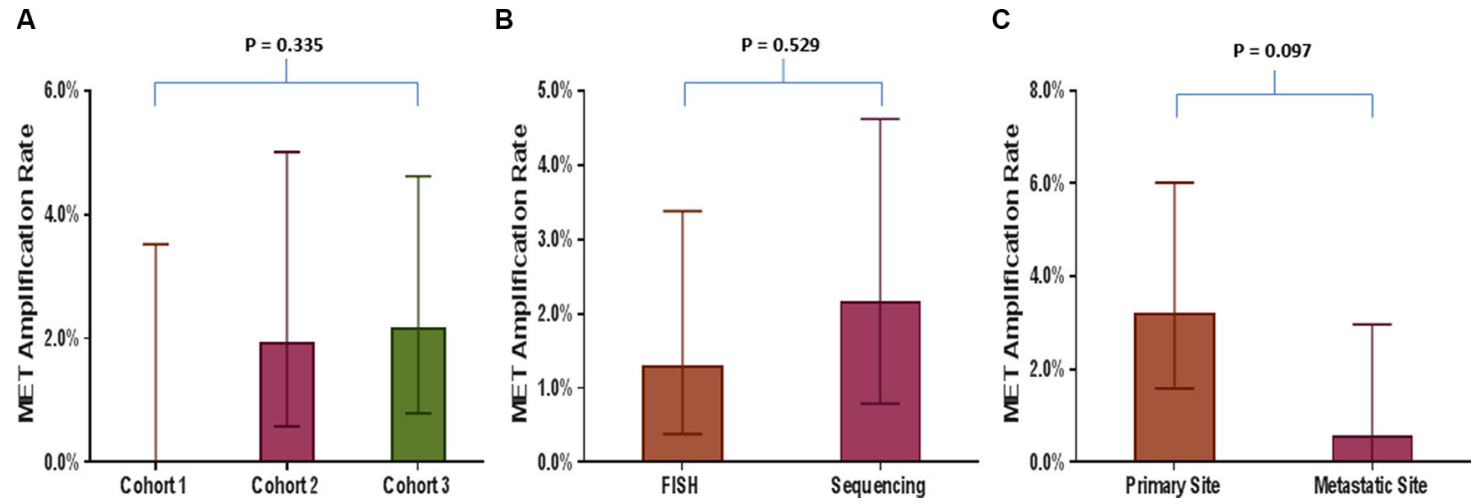

Figure 1: Comparison of MET amplification rate in various tumor tissue based analyses. Bar graphs comparing MET amplification rate between (A) Different cohorts of patients with tumor tissue-based analyses (cohort 1 vs. 2 vs. 3); (B) Two methodologies used to assess MET amplification, fluorescence in situ hybridization (FISH) and sequencing; (C) Primary and metastatic site.
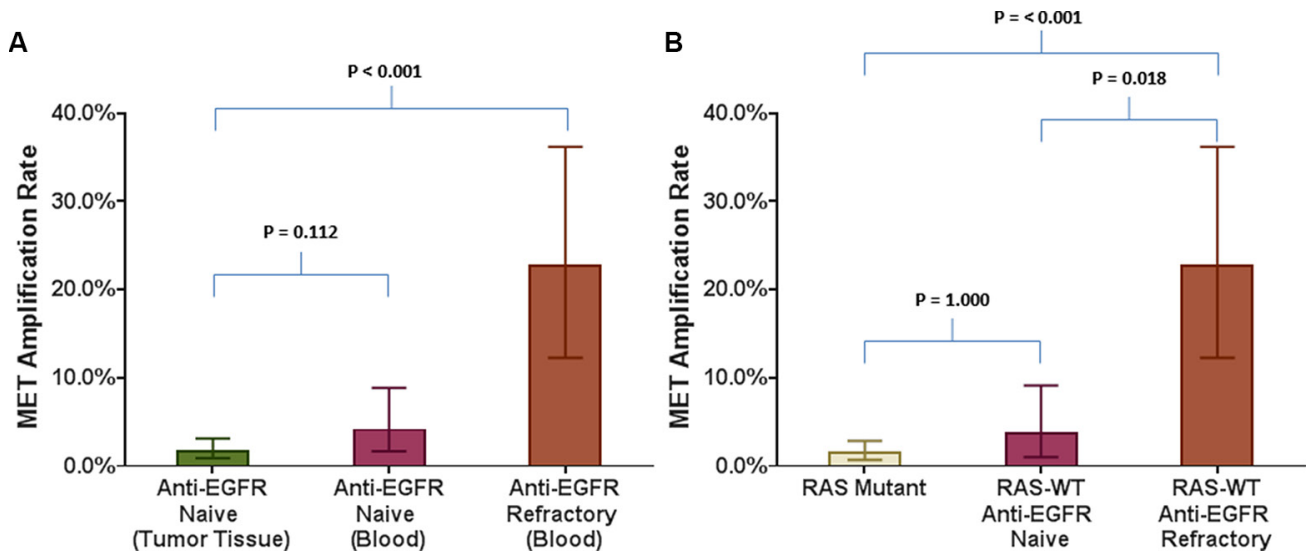

Figure 2: Comparison of MET amplification rate in various tumor tissue based and blood based analyses in relation to refractoriness to anti-EGFR therapy. Bar graphs comparing MET amplification rate between (A) Anti-EGFR naïve tumor tissue biopsies and blood of anti-EGFR refractory RAS wild type patients; (B) Blood from RAS mutant patients and RAS wild type patients who are either anti-EGFR naïve or refractory to anti-EGFR therapy. 
We therefore conclude that MET amplification is a very low prevalence tissue biomarker in de novo $\mathrm{mCRC}$ and therefore a difficult target for enrichment trials with MET targeted therapy. We also recommend FISH or NGS with adequate coverage as ideal tests for assessment of MET amplification in $\mathrm{mCRC}$.

Despite the low prevalence of MET amplification in mCRC, we found that a substantial subset (23\%) of patients with $\mathrm{mCRC}$, who have been treated and are refractory to anti-EGFR antibodies, acquire MET amplification, as detected in cfDNA. Bardelli et al. showed that acquired MET amplification in tumor tissue is associated with acquired resistance to anti-EGFR antibodies in patients of mCRC. [8] Our findings indicate similar phenomenon and suggest the possibility of detecting MET amplification mediated resistance to anti-EGFR therapies using cfDNA. In patients with lung cancer, high level of consistency is seen between peripheral blood and tissue with regards to MET gene amplification. [15] We therefore propose that cfDNA could be a potential blood-based option in lieu of repeated post-treatment tissue biopsies for assessing acquisition of MET amplified phenotype in mCRC. However, it should be noted that additional mutations that could be associated with acquired resistance to anti-EGFR therapy were also seen with MET amplifications in cfDNA (Supplementary Tables S1 and S2). It is uncertain if MET amplification is the sole driver of resistance in these cases, and this heterogeneity may be a barrier to therapeutic interventions to target MET after acquisition of resistance to EGFR inhibition.

Future efforts should focus on refining evaluation of MET amplification. Using FISH for amplification is not without its drawbacks. Since FISH assessed increase in CEP7 copies $>3$ may reflect either true polysomy or centromeric amplification, newer methodologies, such as multiplex ligation-dependent probe amplification that allow simultaneous quantification of multiple loci, can help detect focal MET amplifications that are missed by FISH and may be effective adjunct evaluation in clinical trials. [16] Furthermore, other unique molecular aberration, such as MET exon 14 deletion which can lead to MET overexpression, also need further investigations. [17] Efforts using serial cfDNA analyses are needed to determine whether these acquired MET amplifications are transitory or perpetual. Additionally, to fully comprehend the nature of acquired resistance to anti-EGFR therapy, the role of molecular alterations that may be acquired concurrently with MET amplifications needs to be investigated.

\section{MATERIALS AND METHODS}

\section{Patients}

We performed systematic analyses of $795 \mathrm{mCRC}$ patients, at MD Anderson Cancer Center, Houston, Texas, U.S.A, between January 2010 and September 2015, across
4 different cohorts, who underwent MET amplification testing by various methods. Cohort $1(n=103)$ comprised of newly diagnosed $\mathrm{mCRC}$ patients with resected liver metastases. Cohorts $2(n=208)$ and $3(n=279)$ contained refractory $\mathrm{mCRC}$ patients with pre-treatment tumor tissue biopsies (both primary and metastatic sites). [3] Cohort $4(n=205)$ included $\mathrm{mCRC}$ patients with prospectively collected plasma after at least one-prior line of therapy (Table 1) (see details in eMethods in Supplement).

\section{Samples and MET amplification testing}

In cohort 1 and cohort 2, pre-treatment formalinfixed paraffin embedded (FFPE) tumor tissue was tested using fluorescence in-situ hybridization (FISH). Focal MET amplification was defined as MET/CEP7 ratio $\geq$ 2.0. In cohort 3, pre-treatment FFPE tumor tissue was sequenced with HiSeq (Illumina) with full exome coverage for 202 genes (average depth 800) including MET. MET amplification was defined as $\geq 4$ copies identified by an inhouse algorithm, as previously described. [18] In cohort 4, circulating cell-free DNA (cfDNA) in plasma was analyzed by sequencing on a 54-gene platform optimized for amplifications (Guardant $360^{\circledR}$ ), using methodologies for amplification determination previously reported. [19] All testing was performed in CLIA-certified laboratories (Table 1).

The study was conducted in accordance with the guidelines of the MD Anderson Institutional Review Board.

\section{Statistical analysis}

The primary objective was to determine prevalence of MET amplification in $\mathrm{mCRC}$ in tissue-based and bloodbased biopsies. MET amplification rate was summarized using percentages and 95\% confidence intervals (CIs). Categorical variables were compared using Fisher exact tests. All statistical tests were two-sided, and a $P$ value of 0.05 , when appropriate was considered significant. Statistical analyses were performed using SAS version 9.3 (SAS Institute Inc.).

\section{CONCLUSIONS}

In this large cohort of $\mathrm{mCRC}$, we observed that de novo MET amplification occurs rarely in $\mathrm{MCRC}$, in contrast to previously reported claims. However, acquired MET amplification can be identified by cfDNA in a significant subset of mCRC patients that are refractory to anti-EGFR antibodies. We therefore, conclude that MET amplification appears to play a minor role in de novo colorectal carcinogenesis but may play an important role in acquired anti-EGFR resistance. These findings have clear implications for identifying patient populations and for designing appropriate clinical trials using MET inhibitors in $\mathrm{mCRC}$. 


\section{ACKNOWLEDGMENTS}

None.

\section{CONFLICTS OF INTEREST}

No conflicts of interest.

\section{FINANCIAL SUPPORT}

No financial disclosures.

\section{REFERENCES}

1. Raghav KPS, Eng C. Role of the MET-HGF axis in colorectal cancer: precepts and prospects. Colorectal Cancer. 2012; 1:329-341.

2. Birchmeier C, Birchmeier W, Gherardi E, Vande Woude GF. Met, metastasis, motility and more. Nat Rev Mol Cell Biol. 2003; 4:915-925.

3. Jardim DL, Tang C, Gagliato Dde M, Falchook GS, Hess K, Janku F, Fu S, Wheler JJ, Zinner RG, Naing A, Tsimberidou AM, Holla V, Li MM, et al. Analysis of 1,115 patients tested for MET amplification and therapy response in the MD Anderson Phase I Clinic. Clinical cancer research. 2014; 20:6336-6345.

4. Peters S, Adjei AA. MET: a promising anticancer therapeutic target. Nature reviews Clinical oncology. 2012; 9:314-326.

5. Lennerz JK, Kwak EL, Ackerman A, Michael M, Fox SB, Bergethon K, Lauwers GY, Christensen JG, Wilner KD, Haber DA, Salgia R, Bang YJ, Clark JW, et al. MET amplification identifies a small and aggressive subgroup of esophagogastric adenocarcinoma with evidence of responsiveness to crizotinib. J Clin Oncol. 2011; 29:4803-4810.

6. Lee J, Seo JW, Jun HJ, Ki CS, Park SH, Park YS, Lim HY, Choi MG, Bae JM, Sohn TS, Noh JH, Kim S, Jang HL, et al. Impact of MET amplification on gastric cancer: possible roles as a novel prognostic marker and a potential therapeutic target. Oncol Rep. 2011; 25:1517-1524.

7. Smolen GA, Sordella R, Muir B, Mohapatra G, Barmettler A, Archibald H, Kim WJ, Okimoto RA, Bell DW, Sgroi DC, Christensen JG, Settleman J, Haber DA. Amplification of MET may identify a subset of cancers with extreme sensitivity to the selective tyrosine kinase inhibitor PHA665752. Proc Natl Acad Sci U S A. 2006; 103:2316-2321.

8. Bardelli A, Corso S, Bertotti A, Hobor S, Valtorta E, Siravegna G, Sartore-Bianchi A, Scala E, Cassingena A, Zecchin D, Apicella M, Migliardi G, Galimi F, et al. Amplification of the MET receptor drives resistance to anti-EGFR therapies in colorectal cancer. Cancer discovery. 2013; 3:658-673.

9. Zeng ZS, Weiser MR, Kuntz E, Chen CT, Khan SA, Forslund A, Nash GM, Gimbel M, Yamaguchi Y,
Culliford ATt, D’Alessio M, Barany F, Paty PB. c-Met gene amplification is associated with advanced stage colorectal cancer and liver metastases. Cancer letters. 2008; 265:258-269.

10. Cerami E, Gao J, Dogrusoz U, Gross BE, Sumer SO, Aksoy BA, Jacobsen A, Byrne CJ, Heuer ML, Larsson E, Antipin Y, Reva B, Goldberg AP, et al. The cBio cancer genomics portal: an open platform for exploring multidimensional cancer genomics data. Cancer discovery. 2012; 2:401-404

11. Cancer Genome Atlas N. Comprehensive molecular characterization of human colon and rectal cancer. Nature. 2012; 487:330-337.

12. Jimenez RE, Wallis $T$, Tabasczka P, Visscher DW. Determination of Her-2/Neu status in breast carcinoma: comparative analysis of immunohistochemistry and fluorescent in situ hybridization. Modern pathology. 2000; 13:37-45.

13. Downey L, Livingston RB, Koehler M, Arbushites M, Williams L, Santiago A, Guzman R, Villalobos I, Di Leo A, Press MF. Chromosome 17 polysomy without human epidermal growth factor receptor 2 amplification does not predict response to lapatinib plus paclitaxel compared with paclitaxel in metastatic breast cancer. Clinical cancer research. 2010; 16:1281-1288.

14. Galimi F, Torti D, Sassi F, Isella C, Cora D, Gastaldi S, Ribero D, Muratore A, Massucco P, Siatis D, Paraluppi G, Gonella F, Maione F, et al. Genetic and expression analysis of MET, MACC1, and HGF in metastatic colorectal cancer: response to met inhibition in patient xenografts and pathologic correlations. Clinical cancer research. 2011; 17:3146-3156.

15. Chen D, Xu C, Wu J, Zhang Y, Fang M. A comparison of consistency of detecting c-MET gene amplification in peripheral blood and tumor tissue of nonsmall cell lung cancer patients. Journal of cancer research and therapeutics. 2015; 11:C63-67.

16. Wang T, Amemiya Y, Henry P, Seth A, Hanna W, Hsieh ET. Multiplex Ligation-dependent Probe Amplification Can Clarify HER2 Status in Gastric Cancers with "Polysomy 17". Journal of Cancer. 2015; 6:403-408.

17. Lee J, Ou SH, Lee JM, Kim HC, Hong M, Kim SY, Jang J, Ahn S, Kang SY, Lee S, Kim ST, Kim B, Choi J, et al. Gastrointestinal malignancies harbor actionable MET exon 14 deletions. Oncotarget. 2015; 6:28211-28222. doi: 10.18632/oncotarget.4721.

18. Chen K, Meric-Bernstam F, Zhao H, Zhang Q, Ezzeddine N, Tang LY, Qi Y, Mao Y, Chen T, Chong Z, Zhou W, Zheng X, Johnson A, et al. Clinical actionability enhanced through deep targeted sequencing of solid tumors. Clinical chemistry. 2015; 61:544-553.

19. Lanman RB, Mortimer SA, Zill OA, Sebisanovic D, Lopez R, Blau S, Collisson EA, Divers SG, Hoon DS, Kopetz ES, Lee J, Nikolinakos PG, Baca AM, et al. Analytical and Clinical Validation of a Digital Sequencing Panel for Quantitative, Highly Accurate Evaluation of Cell-Free Circulating Tumor DNA. PLoS One. 2015; 10:e0140712. 